\title{
Full-dimensional quantum scattering calculations on ultracold atom-molecule collisions in magnetic fields: The role of molecular vibrations
}

\author{
Masato Morita, ${ }^{1}$ Jacek Kłos, ${ }^{2, *}$ and Timur V. Tscherbul ${ }^{1, \dagger}$ \\ ${ }^{1}$ Department of Physics, University of Nevada, Reno, Nevada 89557, USA \\ ${ }^{2}$ Department of Chemistry and Biochemistry, University of Maryland College Park, College Park, Maryland 20742, USA
}

(Received 28 April 2020; accepted 26 October 2020; published 30 November 2020)

\begin{abstract}
Rigorous quantum scattering calculations on ultracold molecular collisions in external fields present an outstanding computational problem due to strongly anisotropic atom-molecule interactions that depend on the relative orientation of the collision partners, as well as on their vibrational degrees of freedom. Here, we present the first numerically exact three-dimensional quantum scattering calculations on strongly anisotropic atom-molecule $(\mathrm{Li}+\mathrm{CaH})$ collisions in an external magnetic field based on the parity-adapted total angular momentum representation and a new three-dimensional potential energy surface for the triplet $\mathrm{Li}-\mathrm{CaH}$ collision complex developed using the unrestricted coupled-cluster method with single, double, and perturbative triple excitations and a large quadruple-zeta-type basis set. We find that while the full three-dimensional treatment is necessary for the accurate description of cold $\operatorname{Li}\left(M_{S}=1 / 2\right)+\mathrm{CaH}\left(v=0, N=0, M_{S}=1 / 2\right)$ collisions in a magnetic field, the magnetic resonance density and statistical properties of spin-polarized atom-molecule collisions are not strongly affected by vibrational degrees of freedom, justifying the rigid-rotor approximation used in previous calculations. We observe rapid, field-insensitive vibrational quenching in ultracold $\operatorname{Li}\left(M_{S}=\right.$ $1 / 2)+\mathrm{CaH}\left(v=1, N=0, M_{S}=1 / 2\right)$ collisions, leading to efficient collisional cooling of $\mathrm{CaH}$ vibrations.
\end{abstract}

DOI: 10.1103/PhysRevResearch.2.043294

\section{INTRODUCTION}

The quantum dynamics of ultracold molecular collisions is a focal point of several major avenues of research within the emerging field of ultracold molecular gases [1,2]. In addition to their fundamental importance in chemical physics, molecular collisions and chemical reactions determine many key properties of ultracold molecular gases, such as thermalization rates, collision lifetimes, and controllability with external magnetic fields via magnetic Feshbach resonances. A variety of intriguing quantum phenomena reveal themselves at ultralow temperatures due to the large de Broglie wavelengths of the colliding molecules [3] and the absence of thermal averaging over millions of quantum states [4]. Examples include threshold scattering, shape and orbiting resonances, tunneling under the reaction barrier, quantum statistics, and geometric phase [5], all of which can have profound, largely unexplored, and potentially useful effects on collision rates at ultralow temperatures [2].

Recent experimental studies have explored how electric fields and quantum statistics affect the chemical reaction of

\footnotetext{
*Current affiliations: Department of Physics, Joint Quantum Institute, University of Maryland College Park, College Park, Maryland 20742, USA; Department of Physics, Temple University, Philadelphia, Pennsylvania 19122, USA.

${ }^{\dagger}$ ttscherbul@unr.edu

Published by the American Physical Society under the terms of the Creative Commons Attribution 4.0 International license. Further distribution of this work must maintain attribution to the author(s) and the published article's title, journal citation, and DOI.
}

two $\mathrm{KRb}$ molecules at a temperature of $50 \mathrm{nK}[6-8]$ and observed resonance scattering in cold $\mathrm{He}-\mathrm{NO}[9]$ and $\mathrm{He}^{*}-\mathrm{H}_{2}$ collisions [10]. In addition, rapid progress in molecular laser cooling [11-15] has opened the door to studying ultracold collisions of molecules bearing unpaired electron spins (such as $\mathrm{CaH}, \mathrm{SrF}, \mathrm{CaF}, \mathrm{CaOH}, \mathrm{SrOH}$, and $\mathrm{YbOH}$ ) with trapped ultracold atoms. Sympathetic cooling occurs through elastic (momentum-transfer) collisions when a gas of molecules to be cooled is put in thermal contact with an ultracold atomic ensemble [1,16-18]. Inelastic collisions decrease the efficiency of sympathetic cooling by releasing the energy stored in internal molecular degrees of freedom. Therefore, sympathetic cooling can be expected to be effective when elastic collisions greatly outnumber inelastic collisions, with the ratio of elastic-to-inelastic cross sections $\gamma=\sigma_{\mathrm{el}} / \sigma_{\text {inel }}>100$ [19]. Very recently, sympathetic cooling has been observed experimentally in a trapped mixture of $\operatorname{NaLi}\left(a^{3} \Sigma^{+}\right)$molecules with $\mathrm{Na}\left({ }^{2} \mathrm{~S}\right)$ atoms [20].

These experimental advances strongly motivate rigorous, full-dimensional quantum dynamical calculations on ultracold molecular collisions in the presence of external magnetic fields. When converged with respect to all basisset parameters, such calculations provide a direct connection between the scattering observables and the underlying potential energy surfaces (PESs), which could be used to map out intermolecular interactions $[10,21]$ and to provide direct information on the positions and widths of magnetic Feshbach resonances, which are responsible for quantum chaotic behavior in ultracold molecular collisions [22-24]. Even in the presence of substantial uncertainties in the PESs, quantum scattering calculations can provide important statistical insights into ultracold molecular 
scattering, such as the probability distributions of collision rates [24].

However, rigorous quantum scattering calculations on ultracold atom-molecule collisions of current experimental interest (such as $\mathrm{Li}+\mathrm{CaH}, \mathrm{Rb}+\mathrm{SrF}$, and $\mathrm{Rb}+\mathrm{CaF}$ ) pose a challenging computational problem due to strong and anisotropic atom-molecule interactions, which couple a large number of rotational states, leading to very large, computationally intractable systems of coupled-channel (CC) Schrödinger equations. The rotational basis set convergence problem can be partially overcome by using the computationally efficient total angular momentum representation for molecular collisions in external fields [25,26], which allows for converged computations with hundreds of rotational states $[18,27]$. To save computational effort, these calculations kept the internuclear distance of the diatomic molecule fixed at its equilibrium value (the rigid rotor approximation), thereby neglecting the vibrational degrees of freedom (DOF).

Vibrational DOF are unique to molecular species and can affect ultracold collision dynamics by causing vibrational relaxation, inducing shape and Feshbach resonances [28], and modifying chemical reaction probabilities [28,29]. Kozyryev et al. measured the cross section for vibrational relaxation of the first excited $\mathrm{SrOH}$ stretching mode $(1,0,0)$ in cold collisions with $\mathrm{He}$ atoms at $2 \mathrm{~K}$ and found it to be 700 times smaller than the diffusion cross section [30]. A high ratio of momentum transfer to vibrational relaxation cross sections $\left(\gamma=10^{4}\right)$ was measured for cold $\mathrm{He}+\operatorname{ThO}(v=1, N=0)$ collisions [31]. Cold two-body collisions and three-body recombination of large molecules such as naphthalene, stilbene, and benzonitrile may be affected by their vibrational DOF [32-35]. Most recently, the prospects for laser cooling of complex polyatomic molecules with numerous vibrational modes have been investigated [36,37]. Understanding the role of vibrational DOF in ultracold collisions of such molecules is a prerequisite to efficient sympathetic cooling.

Previous theoretical work has explored the effects of molecular vibrations on ultracold atom-molecule collisions in the absence of external fields using converged CC quantum scattering calculations. Balakrishnan et al. [28] studied ultracold $\mathrm{He}+\mathrm{H}_{2}$ collisions and observed rapid enhancement of vibrational relaxation with increasing degree of vibrational excitation of $\mathrm{H}_{2}$. Balakrishnan and Dalgarno [38] and Volpi and Bohn [39] found that vibrational and fine-structure relaxation in ultracold $\mathrm{He}+\mathrm{O}_{2}$ collisions occur slowly, suggesting the possibility of buffer gas cooling and magnetic trapping of vibrationally excited $\mathrm{O}_{2}$ molecules. Similar conclusions were reached by Balakrishnan, Krems, and Groenenboom in their study of cold $\mathrm{He}+\mathrm{CaH}\left({ }^{2} \Sigma\right)$ collisions [40]. More recent studies explored full-dimensional quantum dynamics of $\mathrm{CO}$ $+\mathrm{H}_{2}$ collisions [41] and found large vibrational cooling rates in cold $\mathrm{Ca}+\mathrm{BaCl}^{+}$collisions [42].

Here, we report on the first rigorous, full-dimensional quantum scattering calculation on an atom-molecule collision in the presence of an external magnetic field, using $\mathrm{Li}+\mathrm{CaH}$ as a representative example. The triplet $\mathrm{Li}-\mathrm{CaH}$ interaction is strongly anisotropic [17] with a well depth of $0.7 \mathrm{eV}$, as is typical for polar ${ }^{2} \Sigma$ molecules interacting with alkali-metal atoms [43]. The enormous number of strongly coupled rovibrational basis states of the Li-CaH collision complex has thus far pre- vented numerically exact simulations of quantum dynamics of $\mathrm{Li}+\mathrm{CaH}$ collisions in external fields. In this work, we employ the computationally efficient total angular momentum basis [25] and make explicit use of inversion symmetry to minimize the number of $\mathrm{CC}$ basis states. These improvements allow us to achieve numerical convergence of $\mathrm{Li}+\mathrm{CaH}$ scattering observables using extended rovibrational basis sets including up to 14 vibrational and 56 rotational states of $\mathrm{CaH}$.

Armed with the improved methodology, we explore the collisional properties of an ultracold spin-polarized Li-CaH mixture in the presence of an external magnetic field. In particular, we test the rigid-rotor approximation employed in our previous calculations [18,27] and find it to be qualitatively accurate yet unable to reproduce the quantitative features of collision dynamics. We also study magnetic Feshbach resonances and vibrational energy transfer from the $v=1$ vibrational level of $\mathrm{CaH}$ induced by ultracold collisions with Li atoms. Our calculations reveal rapid, magnetic fieldinsensitive vibrational quenching in ultracold $\mathrm{Li}+\mathrm{CaH}(v=$ $1, N=0$ ) collisions, implying efficient collisional cooling of the vibrational DOF of $\mathrm{CaH}$.

This paper is organized as follows. In Sec. II we present our three-dimensional ab initio PES of the Li-CaH collision complex. In Sec. II B we outline the details of quantum scattering calculations of ultracold $\mathrm{Li}+\mathrm{CaH}$ collisions, focusing on the new aspects of including the dependence on the $\mathrm{CaH}$ vibrational coordinate. The results of quantum scattering calculations are presented and discussed in Sec. III, whereas Sec. IV summarizes the main results of this work.

\section{THEORY}

\section{A. Ab initio interaction PES}

As a prerequisite to full-dimensional quantum dynamical calculations of ultracold $\mathrm{Li}+\mathrm{CaH}$ collisions, we have computed a new three-dimensional (3D) ab initio interaction PES for the triplet electronic state of the Li-CaH collision complex. To this end, we carried out state-of-the-art electronic structure calculations of the Li-CaH interaction energy in Jacobi coordinates $(R, r, \theta)$, where $R$ is the distance between $\mathrm{Li}$ and the center of mass of $\mathrm{CaH}, r$ is the bond distance of $\mathrm{CaH}$, and $\theta$ is the angle between the Jacobi vectors $\mathbf{R}$ and $\mathbf{r}$. All of the $a b$ initio calculations of the two-body $\mathrm{CaH}$ and three body $\mathrm{Li}-\mathrm{CaH}$ potentials were performed with the unrestricted coupled-cluster method with single, double, and noniterative triple excitations, UCCSD(T). To include the effects of the $\mathrm{CaH}$ vibrational motion, its internuclear distance was varied from $r=2.27 a_{0}$ to $r=6.62 a_{0}$, which is sufficiently wide to cover the range of $\mathrm{CaH}$ vibrational states $v=0-13$. The value $r=6.62 a_{0}$ is the largest one accessible with the single-reference UCCSD(T) method, as further stretching resulted in an unphysical interaction potential and convergence problems due to the increasingly multireference character of the electronic wave function. We used the same UCCSD(T) method and basis set of quadruple-zeta quality augmented with midbond functions as in our previous two-dimensional (2D) rigid-rotor calculations [17].

Figure 1 shows a series of contour plots of our Li-CaH PES as a function of $R$ and $r$ at fixed values of $\theta$. The PESs are 


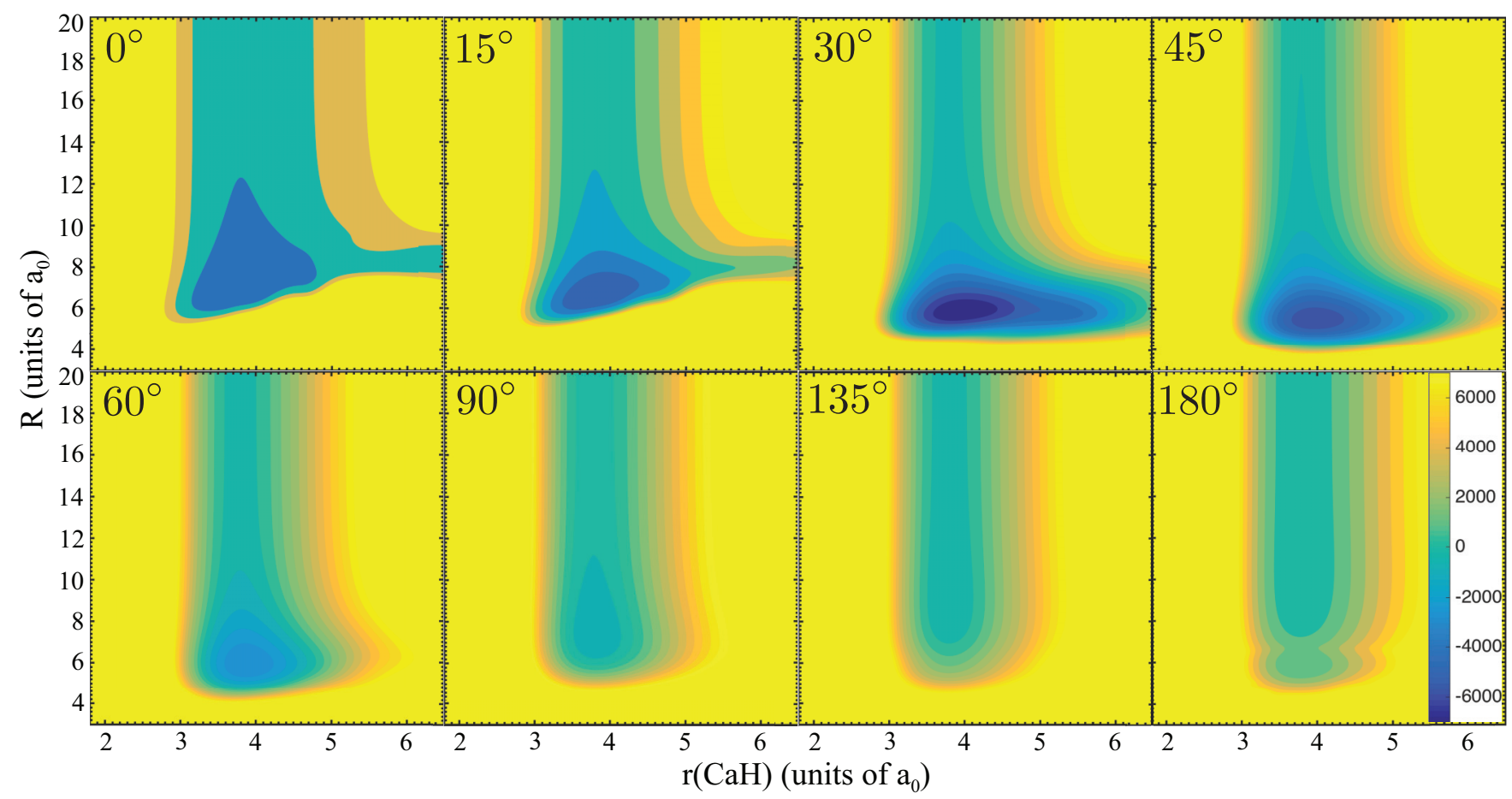

FIG. 1. Contour plot representing the 3D Li-CaH PES of triplet symmetry as a function of the Jacobi coordinates $r, R$, and $\theta$. The global minimum of $-7202.4 \mathrm{~cm}^{-1}$ is located at $r_{e}=3.960 a_{0}, R_{e}=5.804 a_{0}$, and $\theta_{e}=32.5^{\circ}$. The values of the PES are given on the color map (bottom right), in units of $\mathrm{cm}^{-1}$. Lines of the same color represent contours of equal energy.

obtained by adding the 3D interaction potential $V(R, r, \theta)$ to the two-body $\mathrm{CaH}$ potential $V_{\mathrm{CaH}}(r)$. The global minimum of the PES is $7202.4 \mathrm{~cm}^{-1}$ deep and is located at a skewed Li$\mathrm{CaH}$ geometry $\left(r_{e}=3.960 a_{0}, R_{e}=5.804 a_{0}\right.$, and $\left.\theta_{e}=32.5^{\circ}\right)$. The PES becomes increasingly more repulsive with increasing $\theta$ as shown in Fig. 1. We also note that the triplet PES, unlike the singlet one [44], is not reactive within the range of geometries considered.

Our 3D PES is slightly more attractive than the 2D rigidrotor PES developed in our previous calculations [17], which is characterized by $r_{e}=3.803 a_{0}, R_{e}=5.6 a_{0}, \theta=35.0^{\circ}$ and by a well depth of $7063 \mathrm{~cm}^{-1}$. The inclusion of the $\mathrm{CaH}$ stretch pushes the global minimum to larger $R$ by $\simeq 0.2 a_{0}$, increases the equilibrium bending angle by $3^{\circ}$, and deepens the well by $\simeq 160 \mathrm{~cm}^{-1}$.

In this work, we are interested in collisions of $\mathrm{Li}$ and $\mathrm{CaH}$ in their maximally spin-stretched initial states $M_{S_{A}}=$ $M_{S_{B}}=1 / 2$ and $M_{S_{A}}=M_{S_{B}}=-1 / 2$. Neglecting weak spindependent interactions $[44,45]$, the total spin of the collision complex $S$ remains a good quantum number, so we only need to consider the Li-CaH PES of the triplet symmetry $(S=1)$.

\section{B. Quantum scattering theory and computational methodology}

Our full-dimensional quantum scattering calculations are based on the exact solution of the time-independent Schrödinger equation for $\mathrm{a}^{2} \Sigma^{+}$molecule $(A)$ colliding with a ${ }^{2} \mathrm{~S}$ atom $(B)$ in an external magnetic field. The details of the theoretical formalism and its numerical implementation were given in our previous work [17,18,24,27], so we omit the material already covered there and focus on the aspects of the quantum scattering methodology pertinent to vibrational DOF.

The atom-molecule collision complex is described by the Hamiltonian

$$
\hat{\mathcal{H}}=-\frac{1}{2 \mu R} \frac{d^{2}}{d R^{2}} R+\frac{\hat{L}^{2}}{2 \mu R^{2}}+\hat{\mathcal{H}}_{A}+\hat{\mathcal{H}}_{B}+\hat{\mathcal{H}}_{\text {int }},
$$

where $R$ is the atom-molecule distance and $\hat{L}=\hat{J}-\hat{N}-$ $\hat{S}_{A}-\hat{S}_{B}$ is the orbital angular momentum of the collision complex with reduced mass $\mu$ expressed through its total angular momentum $\hat{J}$, the molecular rotational angular momentum $\hat{N}$, and the atomic and molecular electron spin angular momenta $\hat{S}_{i}(i=A, B)$. The Hamiltonian of the vibrating ${ }^{2} \Sigma^{+}$diatomic molecule is

$$
\hat{\mathcal{H}}_{A}=-\frac{1}{2 \mu_{A} r} \frac{d^{2}}{d r^{2}} r+\frac{\hat{N}^{2}}{2 \mu_{A} r^{2}}+V_{A}(r)+\gamma_{\mathrm{sr}} \hat{N} \cdot \hat{S}_{A}+\hat{H}_{A Z},
$$

where $\mu_{A}$ is the reduced mass of the molecule, $r$ is the internuclear distance, $V_{A}(r)$ is the potential energy curve for the $X^{2} \Sigma^{+}$molecular ground state, and $\gamma_{\mathrm{sr}}$ is the spin-rotation constant. The interaction of collision partners with the external magnetic field is described by the corresponding Zeeman Hamiltonians $\hat{H}_{A Z}=g_{e} \mu_{B} \hat{S}_{A_{Z}} B$ and $\hat{\mathcal{H}}_{B Z}=g_{e} \mu_{B} \hat{S}_{B_{Z}} B$, where $\hat{S}_{i_{Z}}$ are the projections of $\hat{S}_{i}$ onto the magnetic field axis, $g_{e} \simeq 2.002$ is the electron $g$-factor, and $\mu_{B}$ is the Bohr magneton. The atomic Hamiltonian $\hat{\mathcal{H}}_{B}=\hat{\mathcal{H}}_{B Z}$ since we neglect the atomic and molecular hyperfine structure known to play a minor role in spin-polarized collisions considered here [18]. The atom-molecule interaction is given by

$$
\hat{\mathcal{H}}_{\text {int }}=\sum_{S, M_{S}} V^{S}(R, r, \theta)\left|S M_{S}\right\rangle\left\langle S M_{S}\right|+\hat{V}_{d d}(\mathbf{R}),
$$


where $V^{S}(R, r, \theta)$ are the 3D interaction PESs for the singlet $(S=0)$ and triplet $(S=1)$ electronic states of the $\mathrm{Li}-\mathrm{CaH}$ complex. Here, we consider ultracold collisions between $\mathrm{Li}$ and $\mathrm{CaH}$ in their fully spin-polarized initial states and we neglect the small matrix elements of the magnetic dipoledipole interaction $\hat{V}_{d d}(\mathbf{R})[24,27,46]$ and of the spin-rotation interaction $\gamma_{\mathrm{sr}} \hat{N} \cdot \hat{S}_{A}$ Eq. (2) between the states of different $S$ values. In this approximation, spin-polarized collisions occur exclusively on a single $S=1$ PES [45] calculated $a b$ initio as described in Sec. II A.

To solve the time-independent Schrödinger equation, we expand the total wave function of the collision complex in a set of spin-rovibrational basis functions [25]

$$
|\Psi\rangle=\frac{1}{R} \sum_{\alpha, J, \Omega} F_{\alpha J \Omega}^{M p}(R)\left|\Phi_{\alpha J \Omega}^{M p}\right\rangle,
$$

where $F_{\alpha J \Omega}^{M p}(R)$ are the radial expansion coefficients and the parity-adapted body-fixed $(\mathrm{BF})$ channel basis states are given by

$$
\begin{aligned}
\left|\Phi_{\alpha J \Omega}^{M p}\right\rangle= & \frac{1}{\sqrt{2}}\left|\chi_{v}^{N}\right\rangle\left[|J M \Omega\rangle\left|N K_{N}\right\rangle\left|S_{A} \Sigma_{A}\right\rangle\left|S_{B} \Sigma_{B}\right\rangle\right. \\
& +(-1)^{X+p}|J M-\Omega\rangle\left|N-K_{N}\right\rangle\left|S_{A}-\Sigma_{A}\right\rangle \\
& \left.\times\left|S_{B}-\Sigma_{B}\right\rangle\right],
\end{aligned}
$$

where $p= \pm 1$ is the inversion parity, $X=J-S_{A}-S_{B}$, and $\left|\chi_{v}^{N}\right\rangle$ are the radial parts of the rovibrational eigenfunctions of the diatomic molecule defined below. The angular basis functions are composed of the eigenstates of $\hat{N}^{2}, \hat{N}_{z}, \hat{S}_{i}^{2}$, and $\hat{S}_{i_{z}}(i=A, B)$, where $K_{N}, \Sigma_{A}$, and $\Sigma_{B}$ (inlcuded in the collective index $\alpha$ ) are the projections of $N, S_{A}$, and $S_{B}$ onto the BF quantization $z$ axis defined by the atom-molecule Jacobi vector $\mathbf{R}[18,25,27,46]$. Further, $|J M \Omega\rangle$ are symmetric top eigenfunctions [47] parametrized by the Euler angles, which specify the orientation of the BF coordinate axes in the spacefixed (SF) frame [25,27], $\Omega$ is the projection of $J$ onto the $\mathrm{BF} z$ axis, and $\Omega=K_{N}+\Sigma_{A}+\Sigma_{B}$. We note that $M$, the projection of $J$ onto the SF quantization axis, is rigorously conserved for collisions in a static magnetic field. Here, we are interested in collisions of spin- $1 / 2$ particles, so both $\Sigma_{A}$ and $\Sigma_{B}$ are nonzero and the normalization factor required in Eq. (5) for $K_{N}=\Sigma_{A}=\Sigma_{B}=0$ can be omitted.

The asymptotic behavior of the radial coefficients $F_{\alpha J \Omega}^{M p}(R)$ defines the scattering $S$ matrix, which provides a complete microscopic description of quantum scattering [48]. To find the coefficients $F_{\alpha J \Omega}^{M p}(R)$, we substitute the CC expansion (4), to the time-independent Schrödinger equation $\hat{H} \Psi=E \Psi$, where $E$ is the total energy, to obtain the $\mathrm{CC}$ equations $[25,49,50]$ :

$$
\begin{aligned}
& {\left[\frac{1}{2 \mu} \frac{d^{2}}{d R^{2}}+E\right] F_{\alpha J \Omega}^{M p}(R)} \\
& \quad=\sum_{\alpha^{\prime}, J^{\prime}, \Omega^{\prime}}\left\langle\Phi_{\alpha J \Omega}^{M p}\left|\frac{\hat{L}^{2}}{2 \mu R^{2}}+\hat{\mathcal{H}}_{A}+\hat{\mathcal{H}}_{B}+\hat{\mathcal{H}}_{\mathrm{int}}\right| \Phi_{\alpha^{\prime} J^{\prime} \Omega^{\prime}}^{M p}\right| F_{\alpha^{\prime} J^{\prime} \Omega^{\prime}}^{M p}
\end{aligned}
$$

The matrix elements over the angular basis functions can be evaluated analytically as described elsewhere [18,25,27,51].
As the first step, we expand the 3D Li-CaH interaction PES in Legendre polynomials

$$
V^{S}(R, r, \theta)=\sum_{\lambda=0}^{\lambda_{\max }} V_{\lambda}^{S}(R, r) P_{\lambda}(\cos \theta),
$$

with $\lambda_{\max }=14$. The explicit inclusion of the vibrational DOF of $\mathrm{CaH} r$ is an essential new element of this work, which distinguishes it from previous atom-molecule scattering calculations in external fields based on the rigid-rotor approximation [17,18,24-27].

The matrix elements over the vibrational basis functions involve the vibrational integrals $[18,25,52]$

$$
\left\langle\chi_{v^{\prime}}^{N^{\prime}}\left|V_{\lambda}^{S}(R, r)\right| \chi_{v}^{N}\right\rangle=\int_{0}^{\infty} \chi_{v^{\prime}}^{N^{\prime}}(r) V_{\lambda}^{S}(R, r) \chi_{v}^{N}(r) d r
$$

where the vibrational basis functions $\left\langle r \mid \chi_{v}^{N}\right\rangle=r^{-1} \chi_{v}^{N}(r)$ in Eq. (5) are solutions of the 1D Schrödinger equation

$$
\left[\frac{-1}{2 \mu_{A}} \frac{d^{2}}{d r^{2}}+\frac{N(N+1)}{2 \mu_{A} r^{2}}+V_{A}(r)\right] \chi_{v}^{N}(r)=E_{v N} \chi_{v}^{N}(r),
$$

where $E_{v N}$ are the rovibrational energy levels of the isolated $\mathrm{CaH}$ molecule.

We solve Eq. (9) numerically for each value of $N$ by diagonalizing the Hamiltonian in the basis of the particle-in-a-box discrete variable representation (sinc-DVR) basis functions [53]. A total of 120 DVR basis functions are employed to obtain converged bound rovibrational levels of $\mathrm{CaH}$ with $v \leqslant 13$. We use the value $\mu_{A}=0.9830336974$ a.m.u. for the reduced mass of $\mathrm{CaH}$. The vibrational integrals in Eq. (8) are preevaluated on a Gauss-Legendre quadrature and stored on hard disk for subsequent use in scattering calculations.

The size of the CC basis set in Eq. (4) is controlled by the cutoff parameters $J_{\max }, v_{\max }$, and $N_{\max }$, which give the maximum values of $J, v$, and $N$. We use $J_{\max }=1, N_{\max }=55$ in all calculations and fix the total angular momentum projection to $M=M_{S_{A}}+M_{S_{B}}$, which corresponds to the incident $s$-wave channel. The convergence of scattering results with respect to $v_{\max }$ is examined in the next section. The total number of scattering channels $N=4634$ for $v_{\max }=13$ and $N=331$ for $v_{\max }=0$. Most of the parameters and physical constants employed in the CC calculations are the same as those used in our previous work [24,25].

The CC equations are solved numerically by means of the Johnson-Manolopoulos log-derivative algorithm [54]. To save computational effort, we separated the radial grid into two regions extending from $R_{\min }=4.0 a_{0}$ to $R_{\text {mid }}=20.0 a_{0}$ (inner region) and from $R_{\text {mid }}$ to $R_{\max }$ (outer region), with the grid spacing of $0.01 a_{0}$ in the inner region and $0.1 a_{0}$ in the outer region. The maximum value of $R_{\max }$ used in the present calculations is $1500 a_{0}$. At $R=R_{\max }$, the log-derivative matrix is transformed from the total angular momentum representation Eq. (4) to a basis set which diagonalizes $\hat{H}_{A}, \hat{\mathcal{H}}_{B Z}$, and the square of the orbital angular momentum operator $\hat{L}^{2}=$ $\left(\hat{J}-\hat{N}-\hat{S}_{A}-\hat{S}_{B}\right)^{2}[18,25]$. The transformed log-derivative matrix is then matched to the scattering boundary conditions to obtain the $S$-matrix and scattering cross sections [18,25]. 


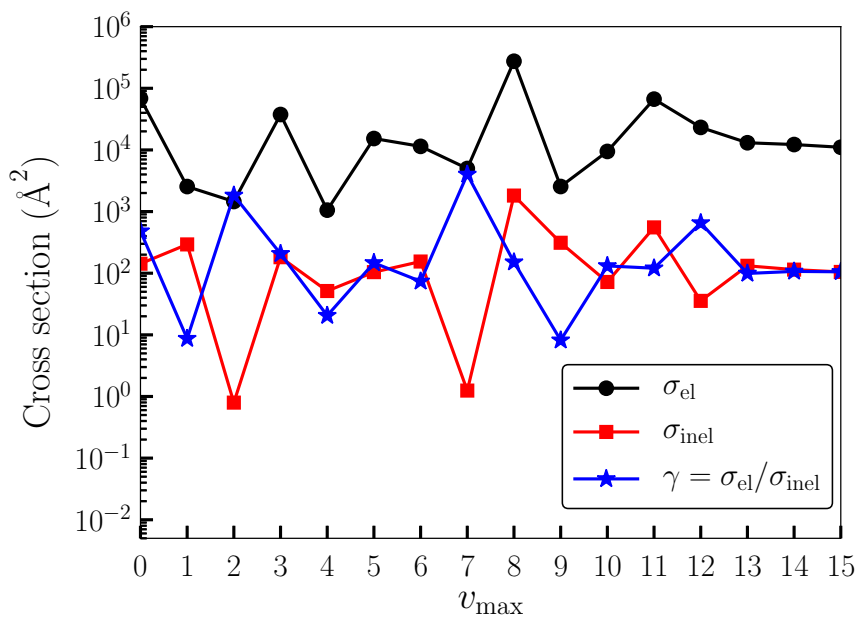

FIG. 2. Convergence of the elastic cross section (black circles), inelastic cross section (red squares), and elastic-to-inelastic ratio (blue stars) for spin-polarized $\mathrm{Li}+\mathrm{CaH}(v=0, N=0)$ collisions with respect to the highest vibrational state of $\mathrm{CaH}$ included in the basis set at the collision energy of $E_{C}=10^{-6} \mathrm{~cm}^{-1}$. The magnitude of the magnetic field is $1000 \mathrm{G}, J_{\max }=1$, and $N_{\max }=55$.

\section{RESULTS}

\section{A. Vibrational basis-set convergence}

To obtain numerically exact collision observables, it is essential to examine their dependence on the CC basis-set size and to demonstrate numerical convergence. Only the results that are converged (or nearly converged) with respect to the basis set correspond to physically meaningful solutions of the Schrödinger equation for a given PES [49,50,55]. We note that basis-set convergence is not required when one is interested in quantities averaged over an ensemble of PESs, such as the cumulative probability distributions [24] examined in Sec. III D below.

Figure 2 shows the elastic and inelastic cross sections as a function of $v_{\max }$, the maximum vibrational state included in the $\mathrm{CC}$ basis (with each vibrational manifold containing 56 rotational states to ensure rotational convergence; see Appendix A). We observe the onset of convergence at $v_{\max }=$ 10 , with both the elastic and the inelastic cross sections reaching their limiting values at $v_{\max } \geqslant 13$. The data shown in Fig. 2 represent the first numerically converged quantum scattering results for a strongly anisotropic atom-molecule collision system in three dimensions in the presence of an external magnetic field.

Significantly, as shown in Fig. 2, it is necessary to include as many as 14 vibrational states $(v=0-13)$ to fully saturate the basis set. Such a slow convergence is due to the strong vibrational anisotropy of the Li-CaH PES illustrated in Fig. 1. Indeed, the bond distance of $\mathrm{CaH}$ near the global minimum structure of the Li-CaH complex is significantly enlarged with respect to the equilibrium bond length, leading to strong couplings between the different vibrational states. A similarly slow convergence is observed with respect to the rotational basis-set size $[17,55]$ due to the strong coupling between the molecular rotational states induced by the angular anisotropy of the PES. All our subsequent calculations will employ the

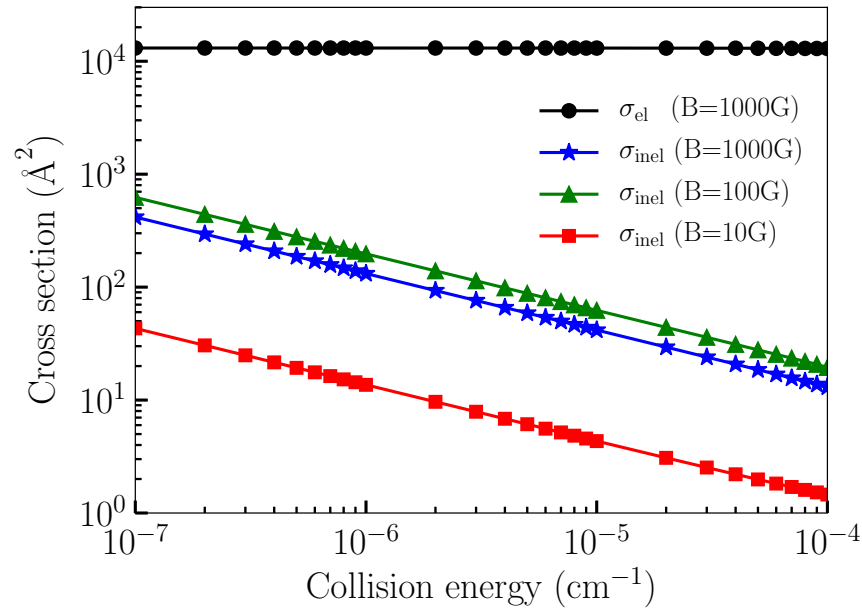

FIG. 3. Collision energy dependence of the elastic cross section (black circles) and inelastic spin-relaxation cross sections for the external magnetic field of $1000 \mathrm{G}$ (blue stars), $100 \mathrm{G}$ (green triangles), and $10 \mathrm{G}$ (red squares) in spin-polarized $\mathrm{Li}-\mathrm{CaH}(v=0$, $N=0)$ collisions. The elastic cross section displays a very weak magnetic-field dependence.

fully converged $v_{\max }=13, N_{\max }=55$ basis unless stated otherwise.

\section{B. Ultracold collision dynamics of $\mathrm{Li}+\mathrm{CaH}(v=0)$ in the ground vibrational state}

We begin by focusing on ultracold collisions of $\mathrm{CaH}$ molecules in their ground electronic and rovibrational state ( $v=0, N=0$ ) with Li atoms in their ground electronic state, with both $\mathrm{Li}$ and $\mathrm{CaH}$ in their maximally spin-stretched Zeeman states $\left(M_{S_{A}}=M_{S_{B}}=1 / 2\right)$. Here, $M_{S_{A}}$ and $M_{S_{B}}$ are the projections of $\hat{S}_{A}$ and $\hat{S}_{B}$ onto the space-fixed quantization axis defined by the external magnetic field. Below, we are interested in elastic (inelastic) collisions, which conserve (change) the values of $M_{S_{A}}$ and $M_{S_{B}}$. As only low-field-seeking states with $M_{S_{i}}=+1 / 2$ can be confined in a magnetic trap, inelastic collisions lead to undesirable trap loss $[1,18]$

Figure 3 shows the cross sections for elastic scattering and inelastic spin relaxation in $\operatorname{Li}\left(M_{S}=1 / 2\right)+\mathrm{CaH}(v=0, N=$ $0, M_{S}=1 / 2$ ) collisions as a function of the collision energy. Following the expected $s$-wave Wigner threshold behavior, the elastic cross sections approach a constant value and the inelastic cross sections scale as $E_{C}^{-1 / 2}$, where $E_{C}$ is the collision energy. We observe that elastic collisions occur much more rapidly than inelastic collisions, indicating good prospects for sympathetic cooling of spin-polarized $\mathrm{CaH}$ molecules with $\mathrm{Li}$ atoms in a magnetic trap. The predominance of elastic over inelastic scattering was also observed in our previous reduced-dimensional calculations on spin-polarized $\mathrm{Li}+\mathrm{CaH}$ collisions [17], suggesting that the rigid-rotor approximation can provide a qualitatively correct picture of spin relaxation and elastic scattering in ultracold collisions of ${ }^{2} \Sigma$ molecules in their ground vibrational states. However, as shown below, the accurate treatment of vibrational DOF is essential for the correct description of the magnetic-field dependence of scattering cross sections, as well as for describing ultracold 


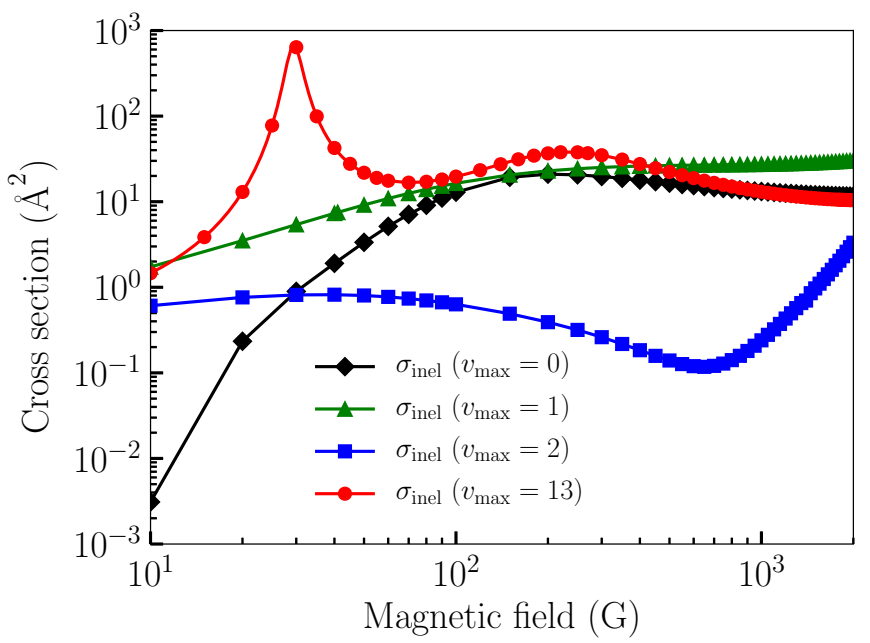

FIG. 4. Magnetic field dependence of inelastic spin-relaxation cross sections in spin-polarized $\mathrm{Li}-\mathrm{CaH}(v=0, N=0)$ collisions at the collision energy of $10^{-4} \mathrm{~cm}^{-1}$ with $v_{\max }=0$ (black diamonds), $v_{\max }=1$ (green triangles), $v_{\max }=2$ (blue squares), and $v_{\max }=13$ (red circles).

collisions of vibrationally excited $\mathrm{CaH}$ molecules with $\mathrm{Li}$ atoms.

In Fig. 4 we plot the magnetic field dependence of the inelastic cross section at a collision energy of $10^{-4} \mathrm{~cm}^{-1}=$ $0.14 \mathrm{mK}$. A pronounced resonance peak is observed at $30 \mathrm{G}$, followed by a broad peak at $B \simeq 300 \mathrm{G}$. To explore whether the resonance peak could be reproduced in calculations using restricted basis sets, we calculated the magnetic-field dependence of the inelastic cross section using basis sets containing only the ground $\left(v_{\max }=0\right)$, the ground and the first excited $\left(v_{\max }=1\right)$, and the lowest three $\left(v_{\max }=2\right)$ vibrational states of $\mathrm{CaH}$. As shown in Fig. 4, no low-field resonances are observed in truncated basis calculations. Furthermore, as shown in Appendix B, the $30 \mathrm{G}$ resonance cannot be reproduced by scaling the interaction PES by a constant factor $\lambda$. This suggests that fully converged 3D calculations are required to map out the magnetic-field dependence of the inelastic cross section. At higher magnetic fields, truncated basis calculations are in better agreement with the fully converged results. In particular, the broad resonance peak at $B \simeq 300 \mathrm{G}$ is well reproduced with only a single vibrational state in the basis $\left(v_{\max }=0\right)$.

We next explore the quantum dynamics of $\mathrm{Li}+\mathrm{CaH}$ collisions with both collision partners in their absolute ground states $\left(M_{S_{A}}=M_{S_{B}}=-1 / 2\right)$. Specifically, we are interested in how the vibrational DOF affect the density of magnetic Feshbach resonances, which could be used to control the scattering length in ultracold atom-molecule mixtures. The statistics of the resonance states determines the degree to which quantum chaos manifests itself in ultracold atom-molecule collisions $[23,56]$ and the probability of complex formation ("sticking") leading to three-body recombination and loss of trapped molecules [22,33].

Figure 5 shows the magnetic field dependence of the $\mathrm{Li}+$ $\mathrm{CaH}$ elastic cross section calculated with different vibrational basis sets for $\mathrm{CaH}$. To locate narrow resonances, we used a
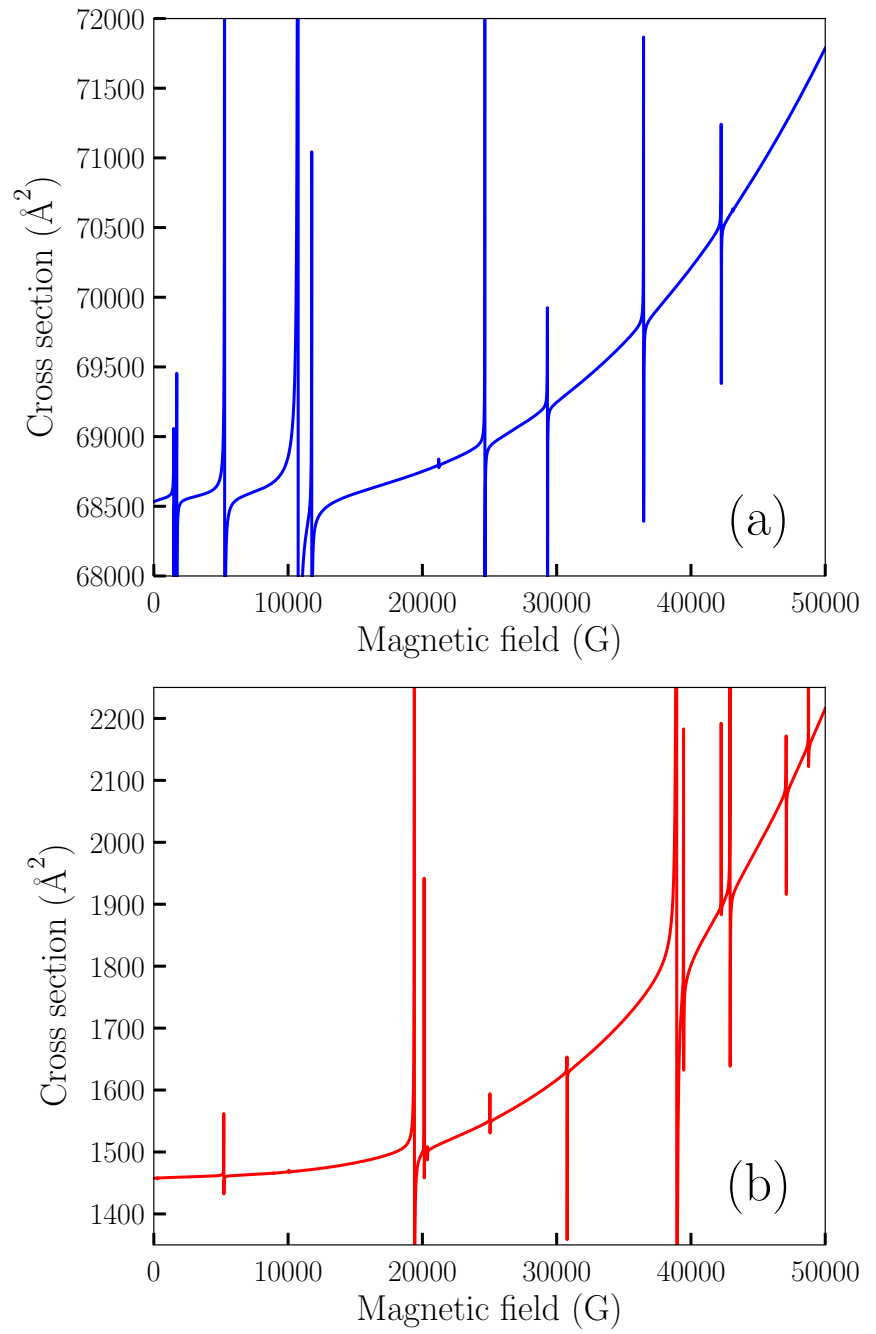

FIG. 5. Magnetic-field dependence of the elastic cross section for $\mathrm{Li}+\mathrm{CaH}(v=0, N=0)$ collisions in the absolute ground Zeeman states at the collision energy of $10^{-6} \mathrm{~cm}^{-1}(1.4 \mu \mathrm{K})$ calculated with (a) $v_{\max }=0$ and (b) $v_{\max }=2$.

dense magnetic field grid of 5001 points spanning the range between 0 and $5 \mathrm{~T}(\Delta B=10 \mathrm{G})$. To make the calculations computationally feasible, we truncated the vibrational basis set to include three lowest-energy vibrational levels of $\mathrm{CaH}$ $\left(v_{\max }=2\right)$. By carrying out fully converged calculations over a limited range of fields $(B=0-0.2 \mathrm{~T})$ we verified that the inclusion of highly excited vibrational states with $v \geqslant 3$ does not qualitatively affect the number of resonances.

Comparing Figs. 5(a) and 5(b), we observe that the number of magnetic Feshbach resonances in $\mathrm{Li}+\mathrm{CaH}$ collisions is fairly low, and it does not change significantly with increasing size of the vibrational basis set. Within the broad range of $B=0-5 \mathrm{~T}$, we observe a total of 11(15) resonances with $\Gamma>$ $10 \mathrm{G}$ for $v_{\max }=0(2)$.

To estimate the mean density of resonances per $1 \mathrm{G}(\bar{\rho})$, we calculated the staircase function $N(B)$ defined as the total number of resonances below the magnetic field $B[23,24]$. Figure 6 shows that the staircase functions calculated for $v_{\max }=0$ to $v_{\max }=2$ are qualitatively similar at $B<4 \mathrm{~T}$. At higher fields the $v_{\max }=2$ staircase function grows more 


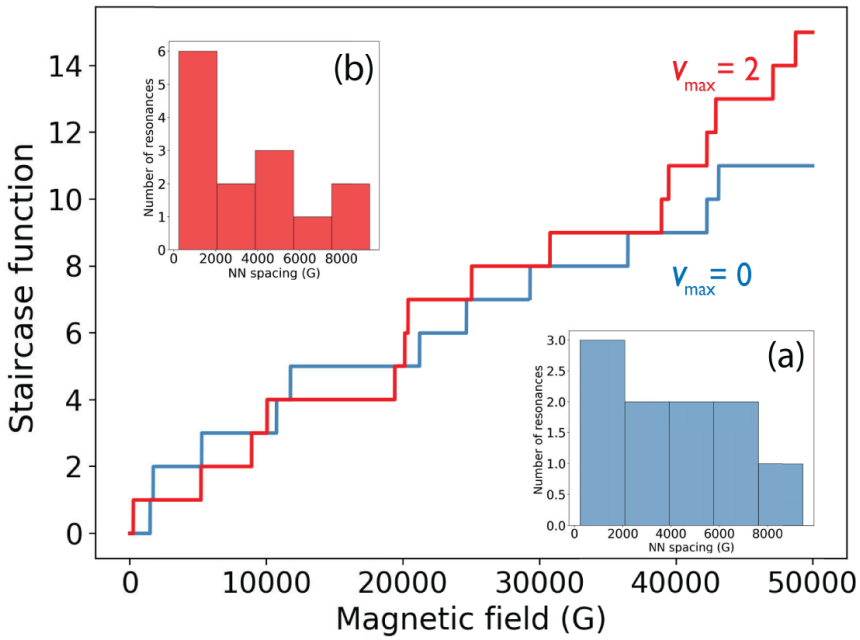

FIG. 6. Magnetic-field dependence of the staircase function $N(B)$ for the resonance spectra shown in Fig. 5 calculated with $v_{\max }=0$ and $v_{\max }=2$. Insets: The corresponding nearest-neighbor spacing distributions are shown in (a) for $v_{\max }=0$ and (b) for $v_{\max }=2$.

rapidly due to the larger number of resonances in this region for $v_{\max }=2$ compared to $v_{\max }=0$ (see Fig. 5). The steplike behavior of the staircase functions indicates that the number of resonances identified in our calculations is insufficient to generate a large enough sample. Thus, the results presented below should be regarded as qualitative estimates, rather than statistically significant predictions.

From a linear fit to $N(B)$ we determine the mean resonance density to be $2.0 \times 10^{-4} \mathrm{G}^{-1}$ for $v_{\max }=0$ and $2.7 \times$ $10^{-4} \mathrm{G}^{-1}$ for $v_{\max }=2$. The resonance density thus increases slightly with the number of vibrational states included in the basis set, although this increase is not nearly as significant as might be expected based on the number of states of the Li$\mathrm{CaH}$ collision complex included in the basis (which increases by a factor of 3 upon going from $v_{\max }=0$ to $v_{\max }=2$ ).

The nearest-neighbor spacing (NNS) distributions of the resonance positions for $v_{\max }=0$ and $v_{\max }=2$ are plotted in the insets in Fig. 6. As with the staircase functions, we observe that the distributions are qualitatively similar, indicating that including more vibrational levels in the basis set does not strongly affect the NNS distributions.

Our results suggest that the mean resonance density is not strongly affected by the vibrational DOF of $\mathrm{CaH}$. It should be noted that intramolecular hyperfine interactions $[44,57,58]$, omitted here, are nearly diagonal in vibrational quantum numbers (at least for low $v$ ). Therefore, while these interactions are likely to increase the overall resonance density, they are unlikely to affect the way in which it is influenced by vibrational DOF.

To understand the physical origin of the low resonance density, we note that the atom-molecule scattering length in the vicinity of a magnetic Feshbach resonance may be written as [59]

$$
a(B)=a_{\mathrm{bg}}\left(1-\frac{\Delta}{B-B_{0}}\right)
$$

where $\Delta=\Gamma_{0} / \delta \mu$ is the zero-energy resonance width expressed via the coupling matrix element between the open and the closed channels,

$$
\Gamma_{0}=2 \pi\left|\left\langle\psi_{c}^{M_{c}}|\hat{W}| \psi_{o}^{M_{o}}\left(E_{0}\right)\right\rangle\right|^{2},
$$

and $\Delta M=M_{o}-M_{c}$ is the difference between the magnetic quantum numbers of the open-channel state $\left|\psi_{o}^{M_{o}}\left(E_{0}\right)\right\rangle$ located above the atom-molecule threshold and the atom-molecule bound state $\left|\psi_{c}^{M_{c}}\right\rangle$. Note that $\Delta M \neq 0$ is required for these states to cross, leading to the appearance of a zero-energy magnetic Feshbach resonance. To couple the states of different $M$, the interaction $\hat{W}$ responsible for the resonance width in Eq. (11) must be spin-dependent.

Here, we are interested in ultracold collisions of ${ }^{2} \Sigma$ molecules ${ }^{2} \mathrm{~S}$ atoms initially in their maximally spin-stretched Zeeman states (either high or low field seeking). In this particular case, the atom-molecule interaction PES is spin independent and does not couple the states of different $M$ values. As a result, magnetic Feshbach resonances can only originate from the intramolecular spin-rotation and intermolecular magnetic dipole-dipole interactions [Eqs. (2) and (3)]. These interactions are diagonal in $v$, so only $v^{\prime}=0$ closed channels are expected to contribute to the magnetic couplings in Eq. (11) that give rise to Feshbach resonances in $\mathrm{Li}+\mathrm{CaH}(v=0, N=0)$ collisions. As a consequence, the density of magnetic Feshbach resonances does not grow dramatically upon the addition of extra vibrational states to the $\mathrm{CC}$ basis set, as shown in Fig. 5. This argument also explains why the resonance density observed in our previous calculations on spin-polarized $\mathrm{Li}+\mathrm{CaH}$ [60], $\mathrm{Li}+\mathrm{SrOH}$ [27], and $\mathrm{Rb}+\mathrm{SrF}[18]$ collisions is nearly as low as in ultracold atomatom collisions despite the large number of closed rotational channels.

Significantly, the above argument implies that the computationally expedient rigid-rotor approximation $[17,18,27]$ can be used to qualitatively predict the spectrum of magnetic Feshbach resonances in ultracold collisions of spin-polarized ${ }^{2} \Sigma$ molecules with alkali-metal atoms. For the $\mathrm{Li}+\mathrm{CaH}$ calculations reported here, the rigid-rotor approximation allows for a 14-fold reduction in the number of scattering channels, decreasing the computational cost by over three orders of magnitude.

We note that the above conclusion does not necessarily apply to the initial atom-molecule states that are not fully spin-polarized. For example, collisions of $\mathrm{CaH}\left(M_{S_{A}}=-1 / 2\right)$ molecules with $\operatorname{Li}\left(M_{S_{B}}=1 / 2\right)$ atoms will be affected by the spin-exchange interaction $J(R, r, \theta) \hat{S}_{A} \cdot \hat{S}_{B}$, with the prefactor $J(R, r, \theta)$ proportional to the difference between the singlet and the triplet PESs in Eq. (3). As the spin-exchange interaction depends explicitly on both the vibrational coordinate $r$ and the spin DOF, it will couple the $v=0$ open channel with the other spin states in the $v^{\prime}>0$ manifolds, potentially leading to significant effects of vibrational DOF on the spectrum of atom-molecule magnetic Feshbach resonances.

\section{Vibrational relaxation in $\mathrm{Li}+\mathrm{CaH}(v=1)$ collisions}

Thus far we have considered the dynamics of spinpolarized collisions of $\mathrm{Li}$ atoms with $\mathrm{CaH}$ molecules in their ground vibrational state. Ultracold collisions of vibrationally 


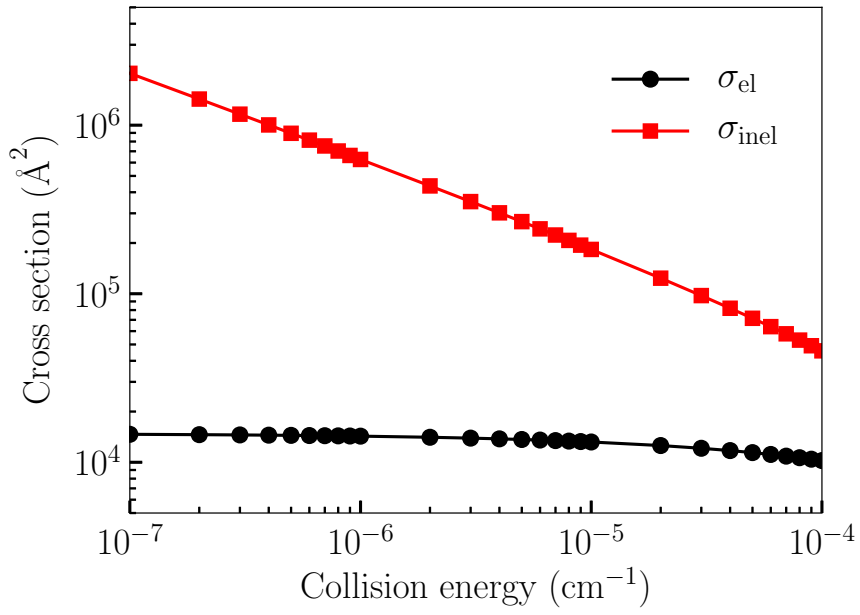

FIG. 7. Elastic (black circles) and total inelastic (red squares) cross sections for ultracold collisions of vibrationally excited $\mathrm{CaH}\left(v=1, N=0, M_{S_{A}}=1 / 2\right)$ with $\operatorname{Li}\left(M_{S_{B}}=1 / 2\right)$ as a function of the collision energy at a magnetic field of $1000 \mathrm{G}$.

excited $\mathrm{CaH}$ molecules with $\mathrm{Li}$ atoms are also of significant interest, as they determine the rate of vibrational cooling of trapped molecules [42]. Rapid vibrational energy transfer in atom-molecule collisions would imply efficient sympathetic cooling of vibrational degrees of freedom to produce trapped molecules in their ground vibrational state.

In Fig. 7 we compare the total elastic and inelastic cross sections for $\mathrm{Li}+\mathrm{CaH}(v=1, N=0)$ collisions in a magnetic field of $1000 \mathrm{G}$. Inelastic relaxation of vibrationally excited $\mathrm{CaH}$ molecules occurs much more rapidly than elastic scattering in the Wigner $s$-wave regime, with $\gamma \ll 1$ at $E_{C}<$ $0.1 \mathrm{mK}$. We verified that the total inelastic cross sections for $\mathrm{Li}+\mathrm{CaH}(v=1, N=0)$ are insensitive to the magnitude of the external magnetic field between 10 and $2000 \mathrm{G}$.

This is in striking contrast with ultracold collision dynamics of ground-vibrational-state $\mathrm{CaH}$ molecules, which tends to be strongly dominated by elastic scattering $(\gamma \gg 1)$ and to vary strongly with the field as shown in Fig. 4 . We attribute the high vibrational relaxation rates to the substantial vibrational anisotropy of the $\mathrm{Li}-\mathrm{CaH}$ interaction PES. As pointed out in Sec. II A, stretching the $\mathrm{CaH}$ vibrational coordinate $r$ results in significant changes in the PES topology, leading to a pronounced $r$ dependence of the Legendre components $V_{\lambda}(r, R)$. This enhances the coupling matrix elements $\left\langle\chi_{v N}\left|V_{\lambda}(r, R)\right| \chi_{v^{\prime} N^{\prime}}\right\rangle$, which drive inelastic transitions between the different vibrational manifolds.

To explore ultracold $\mathrm{Li}+\mathrm{CaH}(v=1)$ scattering in more detail, we examine two key contributions to the total inelastic cross section due to $v$-conserving ("pure") spin relaxation in the $v=1, N=0$ manifold and the $v=1, N=0 \rightarrow v=$ $0, N^{\prime}$ vibrational quenching transitions. Figure 8 shows the inelastic cross sections resolved over final rovibrational states. We observe that vibrational quenching accounts for over 99.99\% of the total inelastic cross section. That is, most of the products of collision-induced vibrational quenching of $\mathrm{CaH}\left(v=1, N=0, M_{S_{A}}=1 / 2\right)$ form in the ground vibrational state. The vibrationally elastic spin relaxation cross section in the $v=1$ spin manifold is comparable in magni- (a)

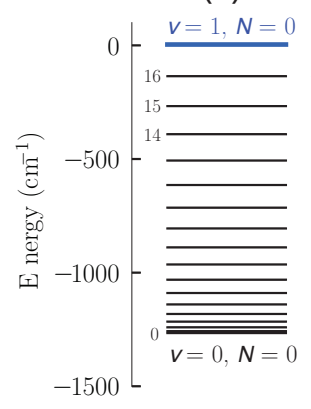

(b)

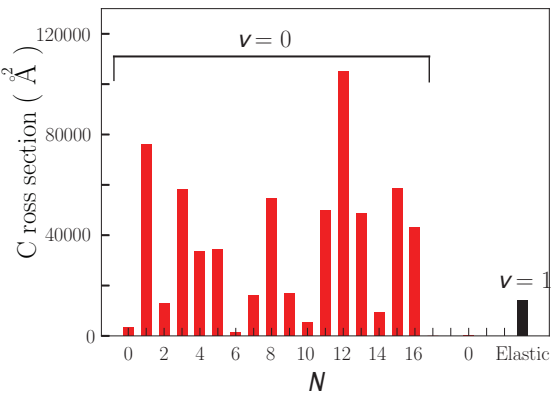

FIG. 8. (a) Rovibrational energy levels of $\mathrm{CaH}\left({ }^{2} \Sigma^{+}\right)$. The energies are shown relative to the rovibrational energy of the $v=1$, $N=0$ state at $B=0$. (b) Rotational product-state distributions following vibrational quenching of $\mathrm{CaH}\left(v=1, N=0, M_{S_{A}}=1 / 2\right)$ by $\mathrm{Li}\left(M_{S_{B}}=1 / 2\right)$ at a collision energy of $1.4 \mu \mathrm{K}$ and magnetic field of $1000 \mathrm{G}$. The following basis-set parameters were used in the calculations: $J_{\max }=1, N_{\max }=55, v_{\max }=13$.

tude to the $v=0$ spin relaxation cross section (see Sec. III B above). The product rotational state distributions of $\mathrm{CaH}(v=$ 0 ) show significant structure, with the population of the $N=0$ and $N=6$ rotational states being strongly suppressed and that of $N=1$ and $N=12$ strongly enhanced. We verified that these results cannot be described by the chaotic Poisson distribution found for the product rotational state distributions of the $\mathrm{K}+\mathrm{KRb}$ chemical reaction [61]. The lack of chaoticity can be attributed to a small number of product rovibrational states of $\mathrm{CaH}(v=0)$ available due to its large rotational constant (17, compared to 142 for $\mathrm{K}+\mathrm{KRb}$ ).

The cross section for $v$-conserving spin relaxation in $\mathrm{Li}+\mathrm{CaH}(v=1, N=0)$ collisions $\sigma_{\text {inel }}^{v=1}$ can be obtained by summing the cross sections for the $\operatorname{Li}\left(M_{S_{A}}=1 / 2\right)+$ $\mathrm{CaH}\left(v=1, N=0, M_{S_{B}}=1 / 2\right) \quad \rightarrow \quad \operatorname{Li}\left(M_{S_{A}}^{\prime}\right)+\mathrm{CaH}(v=$ $1, N=0, M_{S_{B}}^{\prime}$ ) transitions over all final $M_{S_{A}}^{\prime} \neq M_{S_{A}}$ and $M_{S_{B}^{\prime}} \neq M_{S_{B}}$. We obtain $\sigma_{\text {inel }}^{v=1}=2.41 \AA^{2}$ or $3.85 \times 10^{-4} \%$ of the total inelastic cross section, which illustrates that $v$ conserving spin relaxation, which flips the spin projections without changing the rovibrational state of the molecule, makes a negligible contribution to the total inelastic cross sections for $\mathrm{Li}+\mathrm{CaH}(v=1, N=0)$ collisions.

\section{Sensitivity to the interaction potential: Cumulative probability distributions}

In the preceding sections, we used computationally intensive quantum dynamics calculations to obtain converged cross sections for ultracold $\mathrm{Li}+\mathrm{CaH}$ scattering in a magnetic field. These calculations are based on a single adiabatic PES, which contains residual inaccuracies due to imperfections of $a b$ initio methods and fitting errors. As is well known, ultracold scattering observables are very sensitive to these inaccuracies, making quantitative predictions extremely difficult [24]. For example, a $1 \%$ change in the PES depth, which is smaller than the typical uncertainty of state-of-the art ab initio interaction potentials (such as the one developed in Sec. II), can modify the elastic and inelastic cross sections by many orders of 
magnitude due to the presence of numerous scattering resonances $[24,55]$.

We have recently developed a probabilistic approach to this problem based on cumulative probability distributions (CPDs) obtained by averaging scattering observables over an ensemble of slightly different interaction PESs [24]. Specifically, the CPD of a scattering observable $\gamma$ (such as the elastic or inelastic cross section or their ratio) is defined as [24]

$$
F(\Gamma)=P(\gamma \leqslant \Gamma) \approx \frac{N(\gamma \leqslant \Gamma)}{N_{\text {tot }}},
$$

where $P(\gamma \leqslant \Gamma)$ is the probability that $\gamma$ is less than $\Gamma$. In the limit of an infinitely large ensemble size, the CPD may be expressed as $F(\Gamma)=\lim _{N_{\text {tot }} \rightarrow \infty} \frac{N(\gamma \leqslant \Gamma)}{N_{\text {tot }}}$, where $N(\gamma \leqslant \Gamma)$ is the number of samples, for which $\gamma<\Gamma$ and $N_{\text {tot }}$ is the ensemble size. Alternatively, $F(\Gamma)=\int_{0}^{\Gamma} p(\gamma) d \gamma$, where $p(\gamma)$ is the probability density function (PDF) of the observable $\gamma$. Given the PDF, the probability of $\gamma$ falling within a specific range of values $P(a<\gamma<b)=\int_{a}^{b} p(x) d x$. For example, the probability that a collision system has a ratio of elastic-toinelastic cross sections in excess of 100 (a favorable condition for sympathetic cooling) is given by $P_{s}=1-F(\Gamma=100)$. Thus, the CPDs could be used to screen atomic and molecular candidates with favorable properties for sympathetic cooling experiments [24]. Significantly, because the CPDs are much less affected by narrow scattering resonances than single-PES results, they do not require complete basis-set convergence and, hence, can be computed much more efficiently by using severely restricted CC basis sets [24].

Our calculations used a sample of $N_{\text {tot }}=201$ interaction PES $V_{i}$ obtained by scaling the original interaction PES (see Sec. II) by a constant factor $\lambda \in 0.95-1.05$ as described in Ref. [24]. This corresponds to a $5 \%$ uncertainty in our ab initio Li-CaH PESs, which is the typical margin of error of modern high-level $a b$ initio methods for triatomic atom-molecule collision complexes [24]. By carrying out CC calculations for every PES $V_{i}$ in the ensemble $\left\{V_{i}\right\}$, we obtain samples of elastic $\left\{\sigma_{\mathrm{el}, i}\right\}$ and inelastic $\left\{\sigma_{\mathrm{inel}, i}\right\}$ cross sections and of their ratio $\left\{\gamma_{i}\right\}$. Finally, we evaluate the number of samples $N(\gamma \leqslant \Gamma)$ for which the relation $\gamma \leqslant \Gamma$ is satisfied as a function of $\Gamma$ and calculate the CPD using Eq. (12).

To explore the effect of vibrational DOF on the CPDs, we plot in Fig. 9 the cumulative probability distributions of the ratio of the elastic-to-inelastic cross sections for $\mathrm{Li}+\mathrm{CaH}$. The CPDs obtained with one, two, and three vibrational states in the basis set are similar to each other and to the $v_{\max }=0$ CPD. This can be explained by analyzing the $\lambda$ dependence of the cross sections [24]. While adding vibrational DOF increases the number of resonances in the cross sections as a function of $\lambda$, these resonances are averaged out when integrated over a sufficiently large interval of $\lambda$. We note that the differences between the CPDs for different $v_{\max }$ values shown in Fig. 9 cannot be regarded as physically significant due to incomplete convergence of scattering observables and a limited PES sample size, which limit the accuracy of the calculated CPDs to $15 \%-20 \%$.

The resulting distributions all predict a high cumulative probability $P(\gamma \geqslant 100) \simeq 80 \%$, which is favorable for atommolecule sympathetic cooling [17]. The same conclusion was

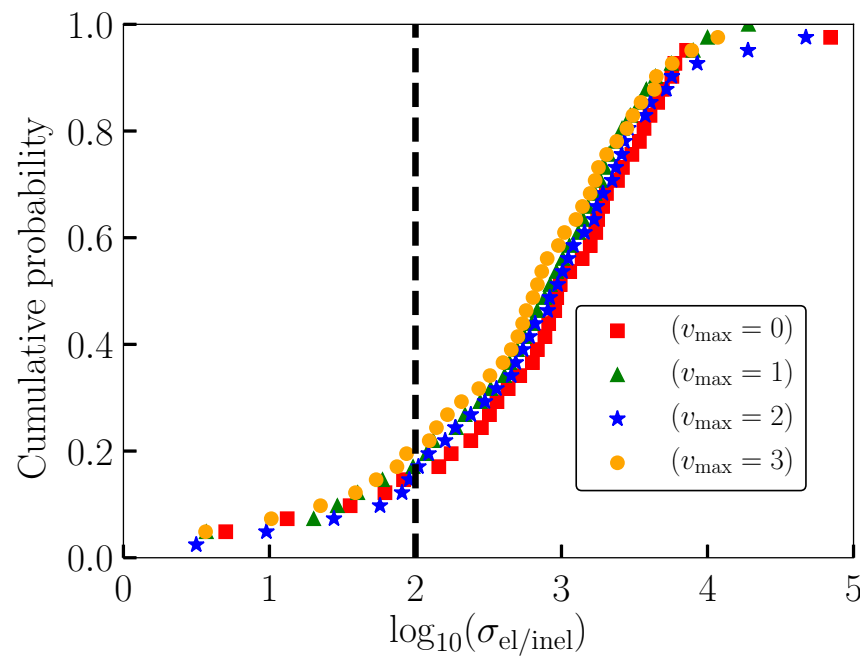

FIG. 9. Cumulative probability distributions of the elastic-toinelastic ratio $\gamma=\sigma_{\text {el }} / \sigma_{\text {inel }}$ for spin-polarized $\mathrm{Li}+\mathrm{CaH}(v=0, N=$ 0 ) collisions calculated with $v_{\max }=0$ (red squares), $v_{\max }=1$ (green triangles), $v_{\max }=2$ (blue stars), and $v_{\max }=3$ (orange circles). The averaging is performed over an ensemble of 201 interaction PESs obtained by multiplying the original 3D PES (Sec. II A) by a constant factor $\lambda=0.95, \ldots, 1.05$. The collision energy is $10^{-4} \mathrm{~cm}^{-1}$ and the magnetic field is $1000 \mathrm{G}$.

reached in our previous work using the rigid-rotor approximation [24], suggesting that the approximation can be applied to describe the statistical properties of ultracold $\mathrm{Li}+\mathrm{CaH}(v=$ $0, N=0)$ collisions.

\section{SUMMARY}

We have presented a computationally efficient methodology, based on the total angular momentum representation [25], for full-dimensional quantum scattering calculations on atom-molecule collisions in the presence of an external magnetic field. We apply the methodology to elucidate the magnetic-field dependence of scattering cross sections for strongly anisotropic collisions of ${ }^{2} \Sigma$ molecular radicals $(\mathrm{CaH})$ with ultracold ${ }^{2} \mathrm{~S}$ atoms (Li). To achieve numerical convergence of scattering observables, we used an extended CC basis set including 14 vibrational and 56 rotational states of $\mathrm{CaH}$ (per each vibrational state), along with the electronic spin states of the collision partners. We developed a new ab initio 3D PES for the triplet state of the $\mathrm{Li}-\mathrm{CaH}$ collision complex featuring an explicit dependence on the $\mathrm{CaH}$ internuclear distance $r$ using a highly correlated UCCSD[T] method and a large basis set. The PES is $7202.4 \mathrm{~cm}^{-1}$ deep and it is also strongly anisotropic.

Our full-dimensional quantum scattering calculations are in qualitative agreement with the previous reduceddimensional $\mathrm{Li}+\mathrm{CaH}$ calculations using the rigid-rotor approximation [17]. Both calculations predict high ratios of elastic-to-inelastic cross sections $\left(\sigma_{\mathrm{el}} / \sigma_{\text {inel }}>100\right)$, indicating favorable prospects for sympathetic cooling of trapped $\mathrm{CaH}$ molecules with $\mathrm{Li}$ atoms. This indicates that the rigid-rotor approximation can provide a qualitatively accurate description 
of ultracold spin-polarized collisions of ${ }^{2} \mathrm{~S}$ atoms with openshell ${ }^{2} \Sigma$-state molecules in their ground vibrational state.

However, a quantitatively accurate description of the inelastic cross sections as a function of the external magnetic field requires the use of a complete rovibrational basis set including 14 vibrational states.

The full-dimensional treatment of the atom-molecule collision problem allowed us to explore vibrational relaxation of $\mathrm{CaH}\left(v=1, N=0, M_{S}=1 / 2\right)$ molecules in ultracold collisions with $\mathrm{Li}$ atoms. We find that vibrational quenching is extremely fast, suggesting that vibrational cooling of $\mathrm{CaH}$ molecules by $\mathrm{Li}$ atoms will be efficient. The quenching cross section is independent of the magnitude of the external magnetic field.

Our calculations uncover the effect of vibrational DOF on the density of magnetic Feshbach resonances in ultracold atom-molecule collisions, an important quantity that determines the extent of quantum chaos, complex formation, and sticking in ultracold molecular collisions [23,62,63]. We find that adding a large number of closed rovibrational channels, while important to achieve numerical convergence, does not significantly alter the mean density of magnetic Feshbach resonances in collisions of spin-polarized atoms and molecules in their absolute ground states. We suggest a tentative explanation for this surprising result: Because magnetic (spin-dependent) couplings in the atom-molecule Hamiltonian are largely independent of $r$, the internuclear distance of the diatomic molecule, closed rovibrational channels with $v^{\prime} \geqslant 1$ remain uncoupled from the $v=0$ incident open channel, dramatically reducing the closed-channel subspace available for the formation of magnetic Feshbach resonances. To rigorously test this hypothesis, it would be desirable to calculate the near-threshold bound states of the collision complex as a function of the external magnetic field and locate their crossings with atom-molecule thresholds, which correspond to zero-energy Feshbach resonances [59].

In future work, we plan to explore ultracold collisions of atoms and molecules in non-fully-spin-polarized initial states. This would require explicit treatment of the low-spin atom-molecule PES of singlet symmetry, which could be realistically accomplished for nonreactive collision systems such as $\mathrm{Rb}+\mathrm{SrF}$ [43] using the total angular momentum representation $[17,18,25,27]$. Such a study could reveal significant vibrational effects on the atom-molecule resonance density due to the $r$ dependence of the Heisenberg exchange interaction. It would also be interesting to explore whether the $r$ dependence of the other spin-dependent interactions (such as the intramolecular spin-rotation interaction in $\mathrm{CaH}$ and/or the intermolecular spin-orbit interaction in $\mathrm{Li}-\mathrm{CaH}$ [64]) could induce magnetic Feshbach resonances in ultracold atom-molecule collisions.

\section{ACKNOWLEDGMENTS}

We are grateful to Dr. Ivan Kozyryev for stimulating discussions. This work was supported by NSF Grant Nos. PHY-1607610 and PHY-1912668 and partially by the U.S. Air Force Office for Scientific Research (AFOSR) under Contract No. FA9550-19-1-0312. J.K. acknowledges financial

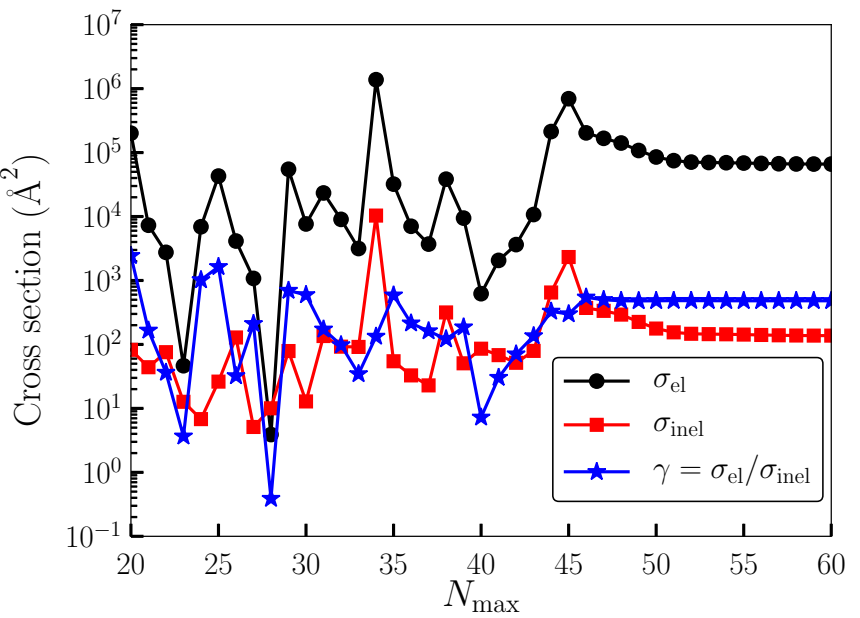

FIG. 10. Elastic cross sections (black circles), inelastic cross sections (red squares), and elastic-to-inelastic ratios (blue stars) for $\mathrm{Li}+\mathrm{CaH}$ as a function of the highest rotational state of $\mathrm{CaH}$ in the basis. Collision energy $E_{C}=10^{-6} \mathrm{~cm}^{-1}$, magnetic field $B=$ $1000 \mathrm{G}, v_{\max }=0$, and $J_{\max }=1$.

support from the U.S. National Science Foundation, Grant No. CHE-1565872 to Millard Alexander as well as XSEDE.org Grant No. CHE-130120 and the University of Maryland High-Performance Deepthought facilities for computational time.

\section{APPENDIX A: ROTATIONAL BASIS-SET CONVERGENCE}

To study rotational basis set convergence, we plot in Fig. 10 the calculated spin relaxation cross section as a function of $N_{\max }$, the number of $\mathrm{CaH}$ rotational states in the $\mathrm{CC}$ basis set containing a single $v=0$ vibrational

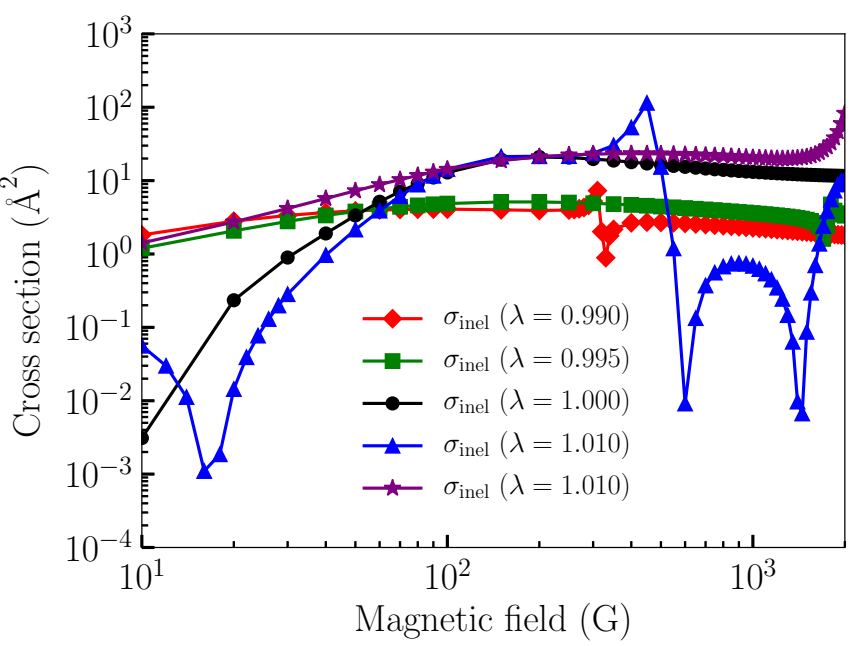

FIG. 11. Magnetic-field dependence of inelastic cross sections for spin-polarized $\mathrm{Li}+\mathrm{CaH}(v=0, N=0)$ collisions at the collision energy of $10^{-4} \mathrm{~cm}^{-1}$ with the interaction potentials scaled by a factor of $\lambda=0.990$ (red diamonds), $\lambda=0.995$ (green squares), $\lambda=1.000$ (black circles), $\lambda=1.005$ (blue triangles), and $\lambda=1.010$ (purple stars). $v_{\max }=0$ and $N_{\max }=55$. 
state. As discussed previously [17], the large anisotropy and depth of the Li-CaH interaction couple a large number of rotational states of $\mathrm{CaH}$. As a result, we observe rapid oscillations of scattering cross sections as a function of $N_{\max }$ up to $N_{\max } \leqslant 45$. A monotonic change in the cross sections continues until $N_{\max } \sim 50$. Interestingly, the elastic-to-inelastic ratio exhibits slightly faster convergence at $N_{\max } \simeq 45$, likely due to correlated residual convergence errors. The requirement of a large $N_{\max }>50$ to obtain convergence is consistent with the results of previous studies using the rigid-rotor approximation $[17,18,24]$. In the calculations reported in the text, we employ $N_{\max }=55$ unless otherwise stated.

\section{APPENDIX B: EFFECT OF PES SCALING}

To explore whether the $30 \mathrm{G}$ resonance in the fully converged 3D results shown in Fig. 4 can be reproduced in truncated $v_{\max }=0$ calculations with a scaled interaction PES, we plot in Fig. 11 the spin relaxation cross sections calculated with several different interaction potentials obtained by multiplying the original PES by a constant scaling factor $\lambda$. We see that while the magnetic-field dependence calculated for $\lambda=1.01$ shows a peak near $50 \mathrm{G}$, the asymmetric shape of the peak is completely different from that of the $30 \mathrm{G}$ resonance shown in Fig. 4. Thus, $\lambda$ scaling of the interaction PES cannot reproduce the resonance features seen in full-dimensional calculations.
[1] L. D. Carr, D. DeMille, R. V. Krems, and J. Ye, Cold and ultracold molecules: Science, technology and applications, New J. Phys. 11, 055049 (2009).

[2] N. Balakrishnan, Perspective: Ultracold molecules and the dawn of cold controlled chemistry, J. Chem. Phys. 145, 150901 (2016).

[3] D. Herschbach, Molecular collisions, from warm to ultracold, Faraday Discuss. 142, 9 (2009).

[4] J. L. Bohn, A. M. Rey, and J. Ye, Cold molecules: Progress in quantum engineering of chemistry and quantum matter, Science 357, 1002 (2017).

[5] B. K. Kendrick, J. Hazra, and N. Balakrishnan, The geometric phase controls ultracold chemistry, Nat. Commun. 6, 7918 (2015).

[6] K. K. Ni, S. Ospelkaus, D. Wang, G. Quéméner, B. Neyenhuis, M. H. G. de Miranda, J. L. Bohn, J. Ye, and D. S. Jin, Dipolar collisions of polar molecules in the quantum regime, Nature (London) 464, 1324 (2010).

[7] S. Ospelkaus, K.-K. Ni, D. Wang, M. H. G. de Miranda, B. Neyenhuis, G. Quéméner, P. S. Julienne, J. L. Bohn, D. S. Jin, and J. Ye, Quantum-state controlled chemical reactions of ultracold KRb molecules, Science 327, 853 (2010).

[8] M. H. G. de Miranda, A. Chotia, B. Neyenhuis, D. Wang, G. Quéméner, S. Ospelkaus, J. L. Bohn, J. Ye, and D. S. Jin, Controlling the quantum stereodynamics of ultracold bimolecular reactions, Nat. Phys. 7, 502 (2011).

[9] S. N. Vogels, J. Onvlee, S. Chefdeville, A. van der Avoird, G. C. Groenenboom, and S. Y. T. van de Meerakker, Imaging resonances in low-energy NO-He inelastic collisions, Science 350, 787 (2015).

[10] A. Klein, Y. Shagam, W. Skomorowski, P. S. Żuchowski, M. Pawlak, L. M. C. Janssen, N. Moiseyev, S. Y. T. van de Meerakker, A. van der Avoird, C. P. Koch, and E. Narevicius, Directly probing anisotropy in atom-molecule collisions through quantum scattering resonances, Nat. Phys. 13, 35 (2016).

[11] J. F. Barry, D. J. McCarron, E. B. Norrgard, M. H. Steinecker, and D. DeMille, Magneto-optical trapping of a diatomic molecule, Nature (London) 512, 286 (2014).

[12] D. J. McCarron, M. H. Steinecker, Y. Zhu, and D. DeMille, Magnetic Trapping of an Ultracold Gas of Polar Molecules, Phys. Rev. Lett. 121, 013202 (2018).
[13] S. Truppe, H. J. Williams, M. Hambach, L. Caldwell, N. J. Fitch, E. A. Hinds, B. E. Sauer, and M. R. Tarbutt, Molecules cooled below the doppler limit, Nat. Phys. 13, 1173 (2017).

[14] I. Kozyryev, L. Baum, K. Matsuda, B. L. Augenbraun, L. Anderegg, A. P. Sedlack, and J. M. Doyle, Sisyphus Laser Cooling of a Polyatomic Molecule, Phys. Rev. Lett. 118, 173201 (2017).

[15] L. Anderegg, B. L. Augenbraun, Y. Bao, S. Burchesky, L. W. Cheuk, W. Ketterle, and J. M. Doyle, Laser cooling of optically trapped molecules, Nat. Phys. 14, 890 (2018).

[16] M. Lara, J. L. Bohn, D. E. Potter, P. Soldan, and J. M. Hutson, Ultracold Rb-OH Collisions and Prospects for Sympathetic Cooling, Phys. Rev. Lett. 97, 183201 (2006).

[17] T. V. Tscherbul, J. Kłos, and A. A. Buchachenko, Ultracold spin-polarized mixtures of ${ }^{2} \Sigma$ molecules with $S$-state atoms: Collisional stability and implications for sympathetic cooling, Phys. Rev. A 84, 040701(R) (2011).

[18] M. Morita, M. B. Kosicki, P. S. Żuchowski, and T. V. Tscherbul, Atom-molecule collisions, spin relaxation, and sympathetic cooling in an ultracold spin-polarized $\mathrm{Rb}\left({ }^{2} \mathrm{~S}\right)-\mathrm{SrF}\left({ }^{2} \Sigma^{+}\right)$mixture, Phys. Rev. A 98, 042702 (2018).

[19] J. P. Burke, Theoretical investigation of cold alkali atom collisions, Ph.D. thesis, University of Colorado (1999).

[20] H. Son, J. J. Park, W. Ketterle, and A. O. Jamison, Collisional cooling of ultracold molecules, Nature 580, 197 (2020).

[21] W. C. Campbell, T. V. Tscherbul, H.-I. Lu, E. Tsikata, R. V. Krems, and J. M. Doyle, Mechanism of Collisional Spin Relaxation in ${ }^{3} \Sigma$ Molecules, Phys. Rev. Lett. 102, 013003 (2009).

[22] J. F. E. Croft and J. L. Bohn, Long-lived complexes and chaos in ultracold molecular collisions, Phys. Rev. A 89, 012714 (2014).

[23] A. Frisch, M. Mark, K. Aikawa, F. Ferlaino, J. L. Bohn, C. Makrides, A. Petrov, and S. Kotochigova, Quantum chaos in ultracold collisions of gas-phase erbium atoms, Nature 507, 475 (2014).

[24] M. Morita, R. V. Krems, and T. V. Tscherbul, Universal Probability Distributions of Scattering Observables in Ultracold Molecular Collisions, Phys. Rev. Lett. 123, 013401 (2019).

[25] T. V. Tscherbul and A. Dalgarno, Quantum theory of molecular collisions in a magnetic field: Efficient calculations based on the total angular momentum representation, J. Chem. Phys. 133, 184104 (2010). 
[26] T. V. Tscherbul, Total angular momentum representation for atom-molecule collisions in electric fields, Phys. Rev. A 85, 052710 (2012).

[27] M. Morita, J. Kłos, A. A. Buchachenko, and T. V. Tscherbul, Cold collisions of heavy ${ }^{2} \Sigma$ molecules with alkali-metal atoms in a magnetic field: $\mathrm{Ab}$ initio analysis and prospects for sympathetic cooling of $\operatorname{SrOH}\left({ }^{2} \Sigma^{+}\right)$by $\operatorname{Li}\left({ }^{2} \mathrm{~S}\right)$, Phys. Rev. A 95, 063421 (2017).

[28] N. Balakrishnan, R. C. Forrey, and A. Dalgarno, Quenching of $\mathrm{H}_{2}$ Vibrations in Ultracold ${ }^{3} \mathrm{He}$ and ${ }^{4} \mathrm{He}$ Collisions, Phys. Rev. Lett. 80, 3224 (1998).

[29] K. Liu, Perspective: Vibrational-induced steric effects in bimolecular reactions, J. Chem. Phys. 142, 080901 (2015).

[30] I. Kozyryev, L. Baum, K. Matsuda, P. Olson, B. Hemmerling, and J. M. Doyle, Collisional relaxation of vibrational states of SrOH with He at 2 K, New J. Phys. 17, 045003 (2015).

[31] Y. S. Au, C. B. Connolly, W. Ketterle, and J. M. Doyle, Vibrational quenching of the electronic ground state in ThO in cold collisions with ${ }^{3} \mathrm{He}$, Phys. Rev. A 90, 032703 (2014).

[32] D. Patterson, E. Tsikata, and J. M. Doyle, Cooling and collisions of large gas phase molecules, Phys. Chem. Chem. Phys. 12, 9736 (2010).

[33] J. Piskorski, D. Patterson, S. Eibenberger, and J. M. Doyle, Cooling, spectroscopy and non-sticking of trans-stilbene and Nile red, Chem. Phys. Chem. 15, 3800 (2014).

[34] S. Putzke, F. Filsinger, J. Küpper, and G. Meijer, Alternatinggradient focusing of the benzonitrile-argon van der waals complex, J. Chem. Phys. 137, 104310 (2012).

[35] Z. Li, R. V. Krems, and E. J. Heller, Collision dynamics of polyatomic molecules containing carbon rings at low temperatures, J. Chem. Phys. 141, 104317 (2014).

[36] M. Li, J. Kłos, A. Petrov, and S. Kotochigova, Emulating optical cycling centers in polyatomic molecules, Commun. Phys. 2, 148 (2019).

[37] J. Kłos and S. Kotochigova, Prospects for laser cooling of polyatomic molecules with increasing complexity, Phys. Rev. Res. 2, 013384 (2020).

[38] N. Balakrishnan and A. Dalgarno, On the quenching of rovibrationally excited molecular oxygen at ultracold temperatures, J. Phys. Chem. A 105, 2348 (2001).

[39] A. Volpi and J. L. Bohn, Molecular vibration in cold-collision theory, Phys. Rev. A 65, 064702 (2002).

[40] N. Balakrishnan, G. C. Groenenboom, R. V. Krems, and A. Dalgarno, The He-CaH $\left({ }^{2} \Sigma^{+}\right)$interaction. II. Collisions at cold and ultracold temperatures, J. Chem. Phys. 118, 7386 (2003).

[41] B. Yang, P. Zhang, X. Wang, P. C. Stancil, J. M. Bowman, N. Balakrishnan, and R. C. Forrey, Quantum dynamics of $\mathrm{CO}-\mathrm{H}_{2}$ in full dimensionality, Nat. Commun. 6, 6629 (2015).

[42] W. G. Rellergert, S. T. Sullivan, S. J. Schowalter, S. Kotochigova, K. Chen, and E. R. Hudson, Evidence for sympathetic vibrational cooling of translationally cold molecules, Nature 495, 490 (2013).

[43] M. B. Kosicki, D. Kedziera, and P. S. Żuchowski, Ab initio study of chemical reactions of cold $\mathrm{SrF}$ and $\mathrm{CaF}$ molecules with alkali-metal and alkaline-earth-metal atoms: The implications for sympathetic cooling, J. Phys. Chem. A 121, 4152 (2017).

[44] T. V. Tscherbul and J. Kłos, Magnetic tuning of ultracold barrierless chemical reactions, Phys. Rev. Res. 2, 013117 (2020).
[45] E. Abrahamsson, T. V. Tscherbul, and R. V. Krems, Inelastic collisions of cold polar molecules in nonparallel electric and magnetic fields, J. Chem. Phys. 127, 044302 (2007).

[46] L. M. C. Janssen, A. van der Avoird, and G. C. Groenenboom, On the role of the magnetic dipolar interaction in cold and ultracold collisions: Numerical and analytical results for $\mathrm{NH}\left({ }^{3} \Sigma^{-}\right)-\mathrm{NH}\left({ }^{3} \Sigma^{-}\right)$, Eur. Phys. J. D 65, 177 (2011).

[47] R. N. Zare, Angular Momentum (Wiley, New York, 1988).

[48] A. Nock, S. Kumar, H. J. Sommers, and T. Guhr, Distributions of off-diagonal scattering matrix elements: Exact results, Ann. Phys. 342, 103 (2014).

[49] D. Secrest, in Atom-Molecule Collision Theory: A Guide for the Experimentalist, edited by R. B. Bernstein (Plenum, New York, 1979), p. 265.

[50] T. V. Tscherbul, in Cold Chemistry: Molecular Scattering and Reactivity Near Absolute Zero, edited by O. Dulieu and A. Osterwalder (Royal Society of Chemistry, London, 2018), p. 276.

[51] R. T. Pack, Space-fixed vs body-fixed axes in atom-diatomic molecule scattering. Sudden approximations, J. Chem. Phys. 60, 633 (1974).

[52] R. V. Krems and A. Dalgarno, Quantum mechanical theory of atom-molecule and molecular collisions in a magnetic field: Spin depolarization, J. Chem. Phys. 120, 2296 (2004).

[53] D. T. Colbert and W. H. Miller, A novel discrete variable representation for quantum mechanical reactive scattering via the S-matrix Kohn method, J. Chem. Phys. 96, 1982 (1992).

[54] D. E. Manolopoulos, An improved log-derivative method for inelastic scattering, J. Chem. Phys. 85, 6425 (1986).

[55] Y. V. Suleimanov and T. V. Tscherbul, Cold NH-NH collisions in a magnetic field: Basis set convergence versus sensitivity to the interaction potential, J. Phys. B 49, 204002 (2016).

[56] M. D. Frye, M. Morita, C. L. Vaillant, D. G. Green, and J. M. Hutson, Approach to chaos in ultracold atomic and molecular physics: Statistics of near-threshold bound states for $\mathrm{Li}+\mathrm{CaH}$ and Li + CaF, Phys. Rev. A 93, 052713 (2016).

[57] T. V. Tscherbul, J. Kłos, L. Rajchel, and R. V. Krems, Fine and hyperfine interactions in cold $\mathrm{YbF}-\mathrm{He}$ collisions in electromagnetic fields, Phys. Rev. A 75, 033416 (2007).

[58] M. L. González-Martínez and J. M. Hutson, Effect of hyperfine interactions on ultracold molecular collisions: $\mathrm{NH}\left({ }^{3} \Sigma^{-}\right)$with $\operatorname{Mg}\left({ }^{1} S\right)$ in magnetic fields, Phys. Rev. A 84, 052706 (2011).

[59] C. Chin, R. Grimm, P. Julienne, and E. Tiesinga, Feshbach resonances in ultracold gases, Rev. Mod. Phys. 82, 1225 (2010).

[60] M. Morita and T. V. Tscherbul, Restricted basis set coupledchannel calculations on atom-molecule collisions in magnetic fields, J. Chem. Phys. 150, 074110 (2019).

[61] J. F. E. Croft, C. Makrides, M. Li, A. Petrov, B. K. Kendrick, N. Balakrishnan, and S. Kotochigova, Universality and chaoticity in ultracold $\mathrm{K}+\mathrm{KRb}$ chemical reactions, Nat. Commun. 8, 15897 (2017).

[62] M. Mayle, B. P. Ruzic, and J. L. Bohn, Statistical aspects of ultracold resonant scattering, Phys. Rev. A 85, 062712 (2012).

[63] M. Mayle, G. Quéméner, B. P. Ruzic, and J. L. Bohn, Scattering of ultracold molecules in the highly resonant regime, Phys. Rev. A 87, 012709 (2013).

[64] M. Warehime and J. Kłos, Nonadiabatic collisions of $\mathrm{CaH}$ with Li: Importance of spin-orbit-induced spin relaxation in spinpolarized sympathetic cooling of $\mathrm{CaH}$, Phys. Rev. A 92, 032703 (2015). 